\title{
Dephasing of solid-state qubits at optimal points
}

\author{
Yuriy Makhlin ${ }^{1,2}$ and Alexander Shnirman ${ }^{1}$ \\ ${ }^{1}$ Institut für Theoretische Festkörperphysik, Universität Karlsruhe, D-76128 Karlsruhe, Germany \\ ${ }^{2}$ Landau Institute for Theoretical Physics, Kosygin st. 2, 117940 Moscow, Russia
}

\begin{abstract}
Motivated by recent experiments with Josephson-junction circuits, we analyze the influence of various noise sources on the dynamics of two-level systems at optimal operation points where the linear coupling to low-frequency fluctuations is suppressed. We study the decoherence due to nonlinear (quadratic) coupling, focusing on the experimentally relevant $1 / f$ and Ohmic noise power spectra. For $1 / f$ noise strong higher-order effects influence the evolution.

PACS numbers: 85.25.Cp, 03.65.Yz, 75.10.Jm
\end{abstract}

For quantum-information processing it is crucial to preserve phase coherence. Recent experiments [1, 2, 3, 4, 5] with Josephson-junction circuits demonstrated longlived coherent oscillations. They showed resolution, sufficient for detailed studies of the dephasing times and decay laws, stressing the need for the theory analysis of the dissipative dynamics of qubits subject to relevant noise sources.

In solid-state systems decoherence is potentially strong due the host of microscopic modes. In Josephson qubits the noise is dominated by material-dependent sources, such as background-charge fluctuations or variations of critical currents and magnetic fields, with power spectrum peaked at low frequencies, often $1 / f$. A further relevant contribution is the electromagnetic noise of the control circuit, typically Ohmic at low frequencies. The $1 / f$ noise appears difficult to suppress and, since the dephasing is dominated by low-frequency noise, it is particularly destructive. On the other hand, Vion et al. 2] showed that the effect of this noise can be substantially reduced by tuning the linear longitudinal qubit-noise coupling to zero. The same strategy, suppressing the linear qubit-detector coupling, was used to minimize the effect of the quantum detector in the off-state. The achieved coherence time was $2-3$ orders of magnitude longer than in earlier experiments.

The $1 / f$ noise (more generally, strong low-frequency noise) plays a major role in many solid-state systems. The long-range correlations in time make the analysis of its effect difficult. In this letter we analyze the dynamics of a qubit subject to singular noise, with a focus on nonlinear coupling and higher-order effects. We also apply the developed formalism to an environment with regular (e.g., Ohmic thermal) noise.

The dynamics of a dissipative two-level system (spin $1 / 2$, qubit) can be described by the Hamiltonian:

$$
\mathcal{H}=-\frac{1}{2}\left(\varepsilon \sigma_{z}+V \sigma_{z}+U \sigma_{x}\right)+H_{\text {bath }}
$$

The longitudinal $(V)$ and transverse $(U)$ fluctuations may result from various microscopic noise sources, described by $H_{\text {bath}}$. In this letter we analyze the dephasing in the situation of quadratic longitudinal coupling,

$$
V=\lambda X^{2}, \quad U=0,
$$

to a source of Gaussian noise $X(t)$, with noise power $S_{X}(\omega)$. Here $X(t)$ is a basic physical quantity (e.g., voltage or magnetic field), which controls the qubit's Hamiltonian [15]. This model is relevant to a Josephson qubit at an optimal point, investigated in recent experiments 2]. To emphasize specific features of this model, we first recall the description of the dissipative qubit dynamics when the noise is either short-correlated or Gaussian longitudinal. Then we motivate model (2) and show that it can describe the effect of both longitudinal and transverse low-frequency noise. We discuss the statistics of $X$ and $V$, and then analyze dephasing in model (2).

For weak short-correlated noise the dynamics is described by the Bloch equations, regardless of the noise statistics. The weak dissipative effects from many uncorrelated time intervals $\sim \tau_{\mathrm{c}}$ (the correlation time) accumulate and the effect of both longitudinal and transverse noise can be described by the markovian Bloch equations. The dissipative rates are given by the golden rule: the $\sigma_{z}$-relaxation rate $1 / T_{1}=S_{U}(\omega=\varepsilon) / 2$ and the rate of dephasing (decay of $\left.\sigma_{x, y}\right) 1 / T_{2}=1 /\left(2 T_{1}\right)+1 / T_{2}^{*}$, the 'pure' dephasing rate $1 / T_{2}^{*}=S_{V}(\omega=0) / 2$ being dominated by low $\omega$. This approach applies for weak noise with a short correlation time $\tau_{\mathrm{c}} \ll T_{1}, T_{2}$.

One can still rely on the lowest order of the cumulant expansion (but beyond the golden rule) for Gaussian longitudinal noise $V(U=0)$, even for long correlations. The coherence $\left\langle\sigma_{-}(t)\right\rangle$ (here $\sigma_{-}=\left(\sigma_{x}-i \sigma_{y}\right) / 2$ ) decays then as

$$
\left|\left\langle\sigma_{-}(t)\right\rangle\right|=\exp \left(-\frac{1}{2} \int \frac{d \omega}{2 \pi} S_{V}(\omega) \frac{\sin ^{2}(\omega t / 2)}{(\omega / 2)^{2}}\right),
$$

where $S_{V}=\frac{1}{2}\left\langle\left\langle[V(t), V(0)]_{+}\right\rangle\right\rangle_{\omega}$ is the noise power. For instance, for a linear coupling, $V(X)=a X$, to a Gaussian-distributed $1 / f$ (flicker) noise $X$, when $T_{2}^{*}$ defined above vanishes, one finds the dephasing law $\exp \left(-a^{2} X_{\mathrm{f}}^{2} t^{2}\left|\ln \left(\omega_{\mathrm{ir}} t\right)\right| / 2 \pi\right)$ (cf. Refs. [6, 7). Here $X_{\mathrm{f}}^{2}$ sets the magnitude of the noise,

$$
S_{X}(\omega)=X_{\mathrm{f}}^{2} /|\omega|
$$


and may depend on the external conditions, such as temperature. The infra-red cutoff $\omega_{\text {ir }}$ may be set, and controlled, by the details of an experiment. For instance, when a measurement of dephasing, performed over a short time $t$, is averaged over many runs, the fluctuations with frequencies down to the inverse of the total signal acquisition time contribute to the phase randomization [7] (this averaging improves the accuracy and is needed to monitor the oscillations of expectation values $1,2,3,4,[5])$. In an echo-type experiment the contribution of slow fluctuations can be suppressed [6]. The controlled frequency of the compensating echo pulses sets $\omega_{\text {ir }}$ (in Ref. $6 \omega_{\text {ir }} \sim 1 / t$ but it can be varied). Here we focus on the case $\omega_{\text {ir }} t \gtrsim 1$.

Typically, in the mentioned regime of linear coupling to $1 / f$ noise dephasing is strong. To increase the coherence time one tunes the system to a point where the linear term vanishes, $a=0$ [2]. The effect of the remaining quadratic coupling is described by the model (2). Moreover, this model can also account for low-frequency transverse fluctuations $U_{\mathrm{lf}}$. Indeed, in the adiabatic approximation we diagonalize (1) to $-\frac{1}{2} \sqrt{(\varepsilon+V)^{2}+U_{\text {lf }}^{2}} \sigma_{z} \approx$ $-\frac{1}{2}\left(\varepsilon+V_{\text {eff }}\right) \sigma_{z}$, where $V_{\text {eff }}=V+U_{\text {lf }}^{2} /(2 \varepsilon)$. Hence as long as the relaxation due to the resonant $(\omega \sim \varepsilon)$ part of $U$ is negligible, it is sufficient to analyze the model (2).

This analysis requires an account of higher orders, and thus the knowledge not only of $S_{V}(\omega)$ but of the full statistics of $V$. The latter depends on the statistics of the basic quantity $X$ as well as on the qubit-noise coupling. In this letter we consider a Gaussian-distributed $X$ (see below), but due to the nonlinear coupling in Eq. (2) the qubit is subject to non-Gaussian fluctuations $V=\lambda X^{2}$. In combination with long correlations of $1 / f$ noise, this shows that further analysis is needed.

The statistics of the low-frequency fluctuations of the basic quantity $X$ deserves further discussion. The assumed Gaussian statistics is generic for noise produced by many microscopic modes, due to the central limit theorem and regardless of the noise mechanism. As for the flicker (' $1 / f$ ') noise in mesoscopic circuits, it may be dominated by only a few modes (bistable fluctuators) or contain comparable contributions of many of those, depending on the sample, and one may expect non-Gaussian resp. Gaussian noise. This was demonstrated in experiments at kHz- and lower frequencies [8]. While the noise at higher frequencies $\mathrm{MHz}-\mathrm{GHz}$, relevant for the dephasing of qubits, is less explored, recent data suggest that it may have the same nature [5, 6, 9]. Here we focus on the analysis of the influence of Gaussian noise $X$; the effect of a few bistable systems was discussed recently in Ref. 10 . Our analysis on one hand, indicates interesting features of decoherence for quadratic coupling; on the other hand, one can consider the qubit as a probe of the noise, thus our findings may help in identifying the noise mechanism.

Analysis of dephasing in model (2). To be definite, we assume that $\lambda>0$; the sign change has no effect on the dephasing laws, but reverses the phase accumulated due to the nonzero average of $V$. We follow the evolution of the off-diagonal entry of the qubit's density matrix $\left\langle\sigma_{-}(t)\right\rangle=\left\langle S^{\dagger} \sigma_{-} S\right\rangle$, with the evolution operator $S=T \exp \left(-\frac{i}{2} \int_{0}^{t} V \sigma_{z} d t^{\prime}\right)$, which yields $\left\langle\sigma_{-}(t)\right\rangle=$ $P(t) e^{i \varepsilon t / \hbar}\left\langle\sigma_{-}(0)\right\rangle$, where

$$
P(t)=\left\langle\tilde{T} \exp \left(\frac{i}{2} \int_{0}^{t} V d t^{\prime}\right) T \exp \left(\frac{i}{2} \int_{0}^{t} V d t^{\prime}\right)\right\rangle,
$$

with averaging over noise realizations. $T$ and $\tilde{T}$ denote time resp. reverse-time ordering; their combination in Eq. (5D) corresponds to the Keldysh-time ordering. Note the same signs in $\tilde{T} \exp$ and $T$ exp, selected by the operator $\sigma_{-}$.

Gaussian approximation. In the lowest-order perturbative analysis one can use Eq. (3) 11]. For Ohmic fluctuations of $X$, with noise power $S_{X}(\omega)=r \omega \operatorname{coth}(\omega / 2 T)$, one finds $S_{X^{2}}(\omega)=\left(r^{2} / 3 \pi\right) \omega\left(\omega^{2}+4 \pi^{2} T^{2}\right) \operatorname{coth}(\omega / 2 T)$ and Eq. (3) yields for weak noise $\lambda r T \ll 1$ the exponential decay with rate

$$
\frac{1}{T_{2}^{*}}=\frac{\lambda^{2}}{2} S_{X^{2}}(\omega=0)=\frac{4 \pi}{3}(\lambda r)^{2} T^{3} .
$$

This dephasing is stronger suppressed by cooling compared to the case of linear coupling, when $1 / T_{2}^{*} \sim T$.

For $1 / f$ noise one finds $S_{X^{2}}=(4 / \pi) X_{\mathrm{f}}^{4} \ln \left|\omega / \omega_{\text {ir }}\right| /|\omega|$ and $P(t)=\exp \left(-\left[\Gamma_{\mathrm{f}} t \ln \left(\omega_{\text {ir }} t\right) / \pi\right]^{2}\right)$, where

$$
\Gamma_{\mathrm{f}}=\lambda X_{\mathrm{f}}^{2} .
$$

Higher orders: results and discussion. Below we analyze effects beyond this Gaussian approximation. For the Ohmic noise we confirm that Eq. (31) holds at all relevant times, yielding the decay rate ([6). In contrast, for the $1 / f$ noise corrections are strong:

$$
\begin{aligned}
|P(t)| & =\left[1+\left(\frac{2}{\pi} \Gamma_{\mathrm{f}} t \ln \frac{1}{\omega_{\mathrm{ir}} t}\right)^{2}\right]^{-1 / 4}, & & \Gamma_{\mathrm{f}} t \ll 1, \\
& =e^{-\Gamma_{\mathrm{f}} t / 2}, & & \Gamma_{\mathrm{f}} t \gg 1 .
\end{aligned}
$$

At short times we find a very slow decay with time scale $\left(\Gamma_{\mathrm{f}} L_{\Gamma}\right)^{-1}$, where $L_{\Gamma} \equiv \ln \left(\Gamma_{\mathrm{f}} / \omega_{\text {ir }}\right)$ and we assume $L_{\Gamma} \gg 1$ (our qualitative results persist down to $L_{\Gamma} \sim 1$ ): At very short times it reduces to $1-\left[\Gamma_{\mathrm{f}} t \ln \left(\omega_{\mathrm{ir}} t\right) / \pi\right]^{2}$ and coincides with the result of the Gaussian approximation. This slow initial decay may be advantageous for application of quantum error correction. At longer times $t \sim\left(\Gamma_{\mathrm{f}} L_{\Gamma}\right)^{-1}$ the decay crosses over to a power law $\propto 1 / \sqrt{t}$. Finally, at the (parametrically longer) time $t \sim \Gamma_{\mathrm{f}}^{-1}$ the decay becomes exponential (9), due to the high-frequency contribution. For large $L_{\Gamma} \gg 1$ a substantial decay occurs already in the range of Eq. (8).

This unusual decay law translates into a peculiar line shape of the transverse spin susceptibility $\chi_{\sigma_{x}}^{\prime \prime}(\omega)=$ $P(\omega-\varepsilon) / 2($ at $T \ll \varepsilon)$, shown in Fig. 1 Eq. (8) gives 


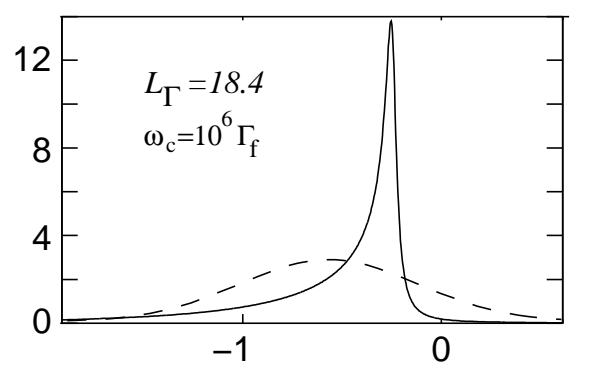

FIG. 1: Line shape $\chi_{\sigma_{x}}^{\prime \prime}(\omega)$, in units of $1 /\left(\Gamma_{\mathrm{f}} L_{\Gamma}\right)$ and as a function of $(\omega-\varepsilon) /\left(\Gamma_{\mathrm{f}} L_{\Gamma}\right)$. Eq. (8) sets a singular universal shape, $\propto(\omega-\varepsilon)^{-1 / 2}$. The high-frequency contribution (9] 12) washes out this singularity on scale $\Gamma_{\mathrm{f}}\left(L_{\Gamma}^{-1}\right.$ in these units) and shifts the peak. The dashed line shows the result of the Gaussian approximation $F_{2}$ combined with the phase shift $F_{1}$.

a singular peak $\propto(\omega-\varepsilon)^{-1 / 2}$, and the term (9) washes it out on the scale $\Gamma_{\mathrm{f}}$, setting the peak height $\sim 1 / \sqrt{\Gamma_{\mathrm{f}}}$. Apart from the dephasing, we find a phase contribution which shifts the peak by $\Gamma_{\mathrm{f}} \ln \left(\omega_{c} / \Gamma_{\mathrm{f}}\right) / \pi$ (if this logarithm exceeds $\ln L_{\Gamma} ; \omega_{c}$ is the unltraviolet cutoff).

Derivation. The Keldysh-time-ordered exponent (5) may be expanded into the linked-cluster series:

$$
P(t)=\exp \left(\sum_{n=1}^{\infty} \frac{1}{n} F_{n}\right),
$$

$F_{n}$ representing the contribution of all connected diagrams of order $n$ in the perturbation $V$ (the Gaussian approximation (3) neglects $F_{n>2}$ ). For the model (2) they are visualized in Fig. 27, with a single cluster in each order. Here the solid lines represent the bare $2 \times 2$ Keldysh Green functions of the bath $\hat{D}=-i\left\langle T_{\mathrm{K}} X(t) X(0)\right\rangle$, whose Keldysh and retarded components [12, 13] are related to the noise power and the response function of the bath, respectively: $D^{\mathrm{K}}=-2 i S_{X}, D^{\mathrm{R}}=-\chi_{X}$. Each vertex contributes a factor $\lambda$ and integration over the time interval $(0 ; t)$. Thus we find in the $n$-th order:

$$
\begin{aligned}
F_{n}(t)= & \frac{(-\lambda)^{n}}{2} \operatorname{tr} \int_{0}^{t} d t_{1} \int_{0}^{t} d t_{2} \ldots \\
& \hat{D}\left(t_{1}-t_{2}\right) \hat{1} \hat{D}\left(t_{2}-t_{3}\right) \hat{1} \ldots \\
= & \frac{(-\lambda)^{n}}{2} \operatorname{tr} \int d \omega_{1} d \omega_{2} \ldots \\
& \hat{D}\left(\omega_{1}\right) \delta_{t}\left(\omega_{1}-\omega_{2}\right) \hat{D}\left(\omega_{2}\right) \delta_{t}\left(\omega_{2}-\omega_{3}\right) \ldots
\end{aligned}
$$

where $\delta_{t}(\omega) \equiv \sin (\omega t / 2) /(\pi \omega)$. The vertices contribute the identity matrices 1 in the Keldysh space, rather than the familiar $\hat{\tau}_{z}$, as a result of the sign structure in Eq. (15); such a perturbation is called 'quantum' 13. For the coupling (2) the averaging in (5) reduces to a Gaussian integral and thus to a determinant of an integral operator. From this standpoint our calculation may be viewed as determinant regularization.

Tractable regimes. The series (10), (11) should be evaluated for each particular time $t$. In general, it is difficult

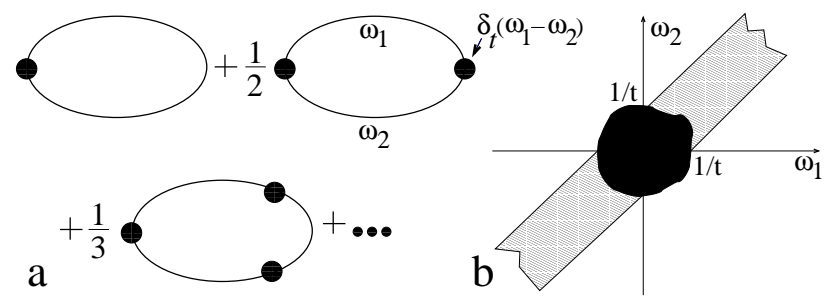

FIG. 2: a. Linked-cluster expansion. The factor $\delta_{t}(\Delta \omega)=$ $\sin (\Delta \omega / 2) /(\pi \Delta \omega)$ at each vertex violates the frequency conservation. b. 'High'- and 'low'-frequency regions dominating the integration in the cluster diagrams.

due to its complex structure: the propagators $\hat{D}$ are diagonal in frequency and the vertices are diagonal in time. However, one can evaluate the dephasing in certain limits: (a) at long times (the exact condition to be specified) $\delta_{t} \approx \delta$, and frequency is conserved at the vertices; (b) at short times the lines become time-indepednent, $\hat{D}\left(t_{i}-t_{i+1}\right) \approx \hat{D}(\Delta t=0)$. These ideas are used below for the case of $1 / f$ noise, when $\hat{D}(\Delta t=0)$ diverges, to find the short- and long-time behavior of $P(t)$.

$1 / f$ noise: (a) higher frequencies. For the $1 / f$ noise it is technically more convenient to discuss the contributions of two frequency ranges at given $t$ (see Fig. 20), rather than the short- and long-time limits.

We begin with higher frequencies: In the integral (11) the adjacent frequencies may differ by $\sim 1 / t$ due to the vertex factors $\delta_{t}$. In the (t-dependent) range $|\omega| \gg 1 / t$ such a shift does not change much the propagators (noise power), and $\delta_{t}(\Delta \omega) \approx \delta(\Delta \omega)$ at each vertex. Thus we find the contribution of high frequencies:

$$
\ln P^{\mathrm{hf}}(t)=-t \int_{\sim 1 / t}^{\infty} \frac{d \omega}{2 \pi} \ln \left(1-2 i \lambda S_{X}(\omega)\right) .
$$

The contribution of frequencies of order $1 / t$ is only estimated by this expression. However, at long times $\Gamma_{\mathrm{f}} t \gg 1$, when Eq. (12) dominates $P(t)$ (as we find below), this contribution is negligible, and we obtain Eq. (9): $\ln \left|P^{\mathrm{hf}}(t)\right|=-\Gamma_{\infty} t$, where

$$
\Gamma_{\infty}=\Gamma_{\mathrm{f}} \int_{0}^{\infty} \frac{d x}{2 \pi} \ln \left(1+\frac{1}{x^{2}}\right)=\frac{1}{2} \Gamma_{\mathrm{f}} .
$$

As for the phase shift, to the logarithmic accuracy in the limit $\ln \left(\Gamma_{\mathrm{f}} t\right) \gg 1$ we find Im $\ln P^{\mathrm{hf}}(t)=\Gamma_{\mathrm{f}} t \ln \left(\Gamma_{\mathrm{f}} t\right) / \pi$.

$1 / f$ noise: (b) lower frequencies. For the analysis of the contribution of low frequencies $|\omega| \ll 1 / t$ one may replace the vertex factor $\delta_{t}(\Delta \omega)$ by its value, $t / 2 \pi$, at $\Delta \omega=0$, and the correlations between frequencies of adjacent lines are irrelevant. Thus the series (10) gives [16]:

$$
\begin{gathered}
F_{n}^{\mathrm{lf}}=\frac{1}{2}\left(\frac{2 i t}{\pi} \int_{\omega_{\mathrm{ir}}}^{\sim 1 / t} \frac{\Gamma_{\mathrm{f}} d \omega}{|\omega|}\right)^{n}=\frac{1}{2}\left(\frac{2}{\pi} i t \Gamma_{\mathrm{f}} \ln \frac{1}{\omega_{\mathrm{ir}} t}\right)^{n},(14) \\
\ln P^{\mathrm{lf}}(t)=-\frac{1}{2} \ln \left(1-\frac{2}{\pi} i t \Gamma_{\mathrm{f}} \ln \frac{1}{\omega_{\mathrm{ir}} t}\right)
\end{gathered}
$$


Again, the contribution of frequencies close to the upper limit, $\omega \sim 1 / t$, is not given reliably by Eq. (15), but it is negligible to the logarithmic accuracy at $\ln \left(1 / \omega_{\text {ir }} t\right) \gg 1$.

The low and high (and intermediate) frequencies contribute at all times, but at short times $\ln |P(t)|$ is dominated by low frequencies and at long times by high frequencies. These leading terms yield Eqs. (8), (9). (The susceptibility $\chi_{X}(\omega)$ enters the analysis, but in the final results we assumed a regular and hence negligible $\chi$.)

Ohmic noise. Let us apply the developed approach to equilibrium thermal noise with quadratic longitudinal coupling, considering the example of an Ohmic bath. Its low-frequency spectral density $\chi_{X}^{\prime \prime}(\omega)=i\left(D^{\mathrm{R}}-D^{\mathrm{A}}\right) / 2=$ $r \omega$ is related by the fluctuation-dissipation theorem to the noise power: $D^{\mathrm{K}}=\left(D^{\mathrm{R}}-D^{\mathrm{A}}\right) \operatorname{coth}(\omega / 2 T)$. The variation scale of the latter is set by temperature, $1 / \tau_{\mathrm{c}} \sim$ $T$. Hence at times $t \gg 1 / T$ changing the frequency by $\sim$ $1 / t$ has little effect on $D^{\mathrm{K}}$, and one may use the long-time approach, with frequency conservation in the diagrams (analysis of $D^{\mathrm{R} / \mathrm{A}}$ does not change this conclusion).

We consider the case of weak-coupling and begin with the lowest order $F_{2}$. Evaluation of this Gaussian cluster involves tr $\hat{D}^{2}=\left(D^{\mathrm{R}}\right)^{2}+\left(D^{\mathrm{A}}\right)^{2}+\left(D^{\mathrm{R}}-\right.$ $\left.D^{\mathrm{A}}\right)^{2}\left[\operatorname{coth}^{2}(\omega / 2 T)-1\right]$. The frequency integral of the first two terms (analytic in the upper/lower half-plane) vanishes, and we find exponential decay with rate (6) at $t \gg 1 / T$ (incl. $t \sim T_{2}^{*}$ ).

As expected for weak noise, the higher orders provide only a small correction to Eq. (6), of order $\left(\lambda r \omega_{\mathrm{c}}\right)^{2}$. Indeed, only the even orders contribute to dephasing, for which tr $\hat{D}^{2 k}=\left(D^{\mathrm{R}}\right)^{2 k}+\left(D^{\mathrm{A}}\right)^{2 k}+$ terms localized at $\omega \lesssim T$. One finds that it is sufficient to evaluate the second-order contribution provided $\lambda \hat{D}(\omega=0) \ll 1$. In the Ohmic case this weak-coupling condition reads $\lambda r T, \lambda r \omega_{\mathrm{c}} \ll 1$.

Higher (non-Gaussian) orders as screening. The exponential decay law (9) appears surprising for $1 / f$ noise, with long-time correlations. In fact, it develops due to the screening of the long-time (low- $\omega$ ) fluctuations, interacting via the term (2), similar to the screening of interaction in the Coulomb gas. Moreover, the calculation of $P(t)$ parallels that of the correlation energy of the Coulomb gas 14]. The result can be found from the lowest-order cluster $F_{2}$ with the solid lines replaced by the properly renormalized (screened) Keldysh propagators $\mathcal{D}$. Diagrammatically this appears natural: the diagrams $F_{n>2}$ in Fig. 22 may be viewed as $F_{2}$ with additional vertices on the lines.

As a result of the screening, qualitatively, the $1 / f$ divergence is cut off at $\omega \sim \Gamma_{\mathrm{f}}$, and the white low- $\omega$ noise produces the exponential decay. We omit the detailed discussion but mention a specific property of the screened $\mathcal{D}$ : unlike the bare $D$ it is $t$-dependent (due to the $t$ dependent vertices). In other words, the screening sets in gradually, with the mentioned saturation at $t \gtrsim \Gamma_{\mathrm{f}}^{-1}$.
Preparation effects. So far we worked under the assumption that at $t=0$ the bath and spin were disentangled and the bath was prepared in the thermal state of $H_{\text {bath }}$. To see, if it can change our conclusions, we considered a typical experiment, in which one monitors the decay of a superposition of the spin states in the presence of permanent spin-bath coupling. We found that for $1 / f$ noise with a non-singular low-frequency susceptibility $\chi(\omega)$ our conculsions about the dephasing persist.

In conclusion, we have analyzed the decay laws of coherence of a qubit coupled quadratically to the environment. We have shown that higher-order effects become important for certain noise spectra (notably, for $1 / f$ ), and found the dephasing times (6)-(9) in various regimes. We are grateful to G. Schön for valuable contributions to this work. We also thank A. Mirlin, K. Gawędzki, D. Esteve, D. Vion, C. Urbina, L. Levitov, P. Degiovanni, Y. Nakamura for fruitful discussions. The work is part of the EU IST Project SQUBIT, the CFN of the DFG, and a research network of the Landesstiftung BW. Y.M. was supported by the Humboldt foundation, the BMBF, and the ZIP programme of the German government.

[1] Y. Nakamura, Yu.A. Pashkin, and J. S. Tsai, Nature 398, 786 (1999), Yu.A. Pashkin et al., ibid. 421, 823 (2003).

[2] D. Vion et al., Science 296, 886 (2002).

[3] Y. Yu et al., Science 296, 889 (2002).

[4] J. M. Martinis et al., Phys. Rev. Lett. 89, 117901 (2002).

[5] I. Chiorescu et al., Science 299, 1869 (2003).

[6] Y. Nakamura et al., Phys. Rev. Lett. 88, 047901 (2002).

[7] A. Cottet et al., in Macroscopic Quantum Coherence and Quantum Computing, edited by D. Averin, B. Ruggiero, and P. Silvestrini (Kluwer/Plenum, NY, 2001), p. 111.

[8] see e.g. A.B. Zorin et al., Phys. Rev. B 53, 13682 (1996), D.J. VanHarlingen et al., unpub. (2003); C.T. Rogers, R.A. Buhrman, Phys. Rev. Lett. 55, 859 (1985).

[9] D. Vion et al., Fortschr. Phys. 51, 462 (2003), R. Simmonds et al., unpublished (2003).

[10] E. Paladino et al., Phys. Rev. Lett. 88, 228304 (2002); Y.M. Galperin et al., cond-mat/0312490.

[11] A. Shnirman, Yu. Makhlin, and G. Schön, Phys. Scr. T102, 147 (2002).

[12] J. Rammer, H. Smith, Rev. Mod. Phys. 58, 323 (1986).

[13] A. Kamenev, in Strongly correlated fermions and bosons in low-dimensional disordered systems, edited by I. Lerner et al. (Kluwer, Dordrecht, 2002).

[14] M. Gell-Mann and K.A. Brueckner, Phys. Rev. 106, 364 (1957), cf. L.S. Levitov, A.V. Shytov Green's functions: theory and practice (MIPT, 1997, in Russian) Ch. 8.

[15] The analysis is easily generalized to several noise sources coupled to arbitrary spin components.

[16] The derivation of Eqs. (12), (15) may involve summation of divergent series. Other methods, e.g., based on functional integration, may be used to justify these results. 